Mayor Paredes, D. y Rodríguez Martínez, D. (2016). Aprendizaje-servicio y práctica docente: una relación para el cambio educativo. Revista de Investigación Educativa, 34(2), 535-552.

DOI: http://dx.doi.org/10.6018/rie.34.2.231401

\title{
Aprendizaje-Servicio y práctica docente: una relación para el cambio educativo*
}

\author{
Service-Learning and Teaching Practice: \\ A Relationship for Change in Education \\ Domingo Mayor Paredes y Dolores Rodríguez Martínez \\ * Universidad de Almería. España. \\ ** Departamento de Educación. Universidad de Almería. España.
}

\begin{abstract}
Resumen
Este artículo presenta los resultados de una investigación centrada en el análisis de la influencia que tienen los proyectos de Aprendizaje-Servicio en el desarrollo y mejora de la práctica escolar. El estudio tomó como eje de indagación un aula de primaria, y los efectos que se iban desencadenando durante la implementación del proyecto en el que participaron: maestra y alumnado de primaria, profesora y alumnado de la universidad y representantes de distintas instituciones. Para ello se utilizó el estudio de caso como metodología de la investigación y la observación participante, entrevistas semiestructuradas y análisis de documentos como estrategias para la recogida de información. Los resultados obtenidos permiten constatar los efectos producidos en relación a las concepciones pedagógicas de la docente, la programación curricular de aula y el desarrollo profesional. A partir de los hallazgos alcanzados se concluye que el Aprendizaje-Servicio abre nuevos horizontes para el diseño de prácticas innovadoras de utilidad social.

Palabras clave: aprendizaje-servicio; aprendizaje por experiencia; innovación pedagógica; desarrollo profesional; estudio de casos.

Correspondencia: Domingo Mayor Paredes. E-mail: lamarencendida@hotmail.com. Dolores Rodríguez Martínez. E-mail: drodri@ual.es. Departamento de Educación. Universidad de Almería.

* El proyecto elegido como unidad de análisis forma parte del programa del Grupo de Investigación para la recuperación de espacios públicos, aprobado por la Dirección General de Innovación Educativa y Formación del Profesorado de la Junta de Andalucía.
\end{abstract}




\begin{abstract}
This article presents the results of a study analyzing the influence of Service-Learning projects on the development and improvement of educational practices. A primary school classroom was used as the study's axis for investigation and the effects that were triggered during the project's implementation where the primary school teacher and students, the university professor and students and representatives of different institutions took part. The research methodology used was a case study and the strategies of collecting information were participant observation, semi-structured interviews and analysis of documents. The results have enabled us to demonstrate the effects of the pedagogical conceptions of the teacher, classroom curricular programming and professional development. Based on these findings, we conclude that Service-Learning opens new horizons for the design of innovative practices of social utility.

Keywords: service-learning; experiential learning; educational innovation; career development; case study.
\end{abstract}

\title{
Introducción
}

Los informes internacionales sobre educación de los últimos años (Delors, 1996; Faure, 1972, OCDE, 2005, etc.), así como estudiosos del ámbito de la educación (Carbonell, 2001; Luengo y Moyá, 2008; Pérez, 2012; Torres, 2011), han señalado, desde distintas posiciones, la importancia de los cambios sociales y la necesidad de avanzar en programas que se sirvan de todas las redes de aprendizaje existentes en la comunidad. Para articular este proceso "hay que dejar de considerar que las diversas formas de enseñanza y aprendizaje son independientes y, [...] en cambio, tratar de realzar el carácter complementario de los ámbitos y periodos de la educación moderna" (Delors, 1996, p. 125).

Los cambios acelerados y la complejidad de las sociedades del siglo XXI han provocado la emergencia de diferentes discursos y prácticas socioeducativas (Ciudades educadoras, Formación en empresas, Comunidades de Aprendizaje, etc.) que quiebran las formas tradicionales de entender las práctica escolares basadas, entre otras cuestiones, en la adquisición de conocimientos de corta vida, primando su valor de cambio, en detrimento del conocimiento con valor de uso que promueve la adquisición de saberes y competencias para conocer e intervenir de forma reflexiva y crítica en la realidad (García y Carmona, 2014; Pérez, 2012). En este marco, adquieren un papel sustantivo las concepciones pedagógicas que orientan las prácticas del profesorado, entendidas como un proceso personal por el cual un individuo estructura su saber a medida que integra los conocimientos que va adquiriendo en diversos espacios de socialización: familia, escuela, comunidad, universidad, medios de comunicación, etc. (Giordan y De Vecchi, 1995). Desde esta perspectiva,

"muchas de las investigaciones realizadas han señalado que las concepciones del profesorado subyacen en sus acciones didácticas y cómo gran parte de los cambios educativos son posibles si los agentes que participan en ellos están dispuestos a modificar sus creencias y sus prácticas" (López-Vargas y Basto-Torrado, 2010, p. 277). 
Quebrar las formas tradicionales de entender la práctica profesional requiere, además, de una reconstrucción del conocimiento práctico de los docentes, donde la teoría debe permitirles analizar y transformar las prácticas (Pérez, 2012); unas prácticas que siguen estando mediatizadas por la aplicación acrítica de prescripciones y normativas legales, así como por los libros de texto como recurso fundamental, olvidando que éstos, sólo ofrecen una interpretación determinada del curriculum oficial conduciendo, de este modo, a la desprofesionalización del docente.

Para favorecer el cambio en las ideas pedagógicas del profesorado se hace necesario generar procesos formativos donde la práctica se convierta en una instancia para el desarrollo profesional, entendido como el conjunto de factores (salario, clima laboral, formación permanente, desarrollo de la autonomía profesional, apertura a nuevos dispositivos por donde circula el saber, etc.) que posibilitan "aumentar la calidad docente, investigadora y de gestión" (Imbernón, 2011, p. 76).

Estas nuevas miradas para afrontar el quehacer educativo constituye uno de los pilares teóricos de las prácticas de Aprendizaje-Servicio (a partir de ahora A-S), entendidas como acciones formativas orientadas a promover aprendizajes significativos, útiles y relevantes en contextos educativos ampliados (escuela-comunidad), donde el profesorado y el estudiantado conforman un grupo de investigación-acción con la intencionalidad de mejorar algunas de las necesidades sentidas en el entorno inmediato. Ello supone un desarrollo del "compromiso ético y social con la comunidad, aprendiendo a aplicar el conocimiento académico a la mejor comprensión y actuación sobre los problemas de la vida cotidiana de su comunidad en todas sus dimensiones, artística, científica, técnica y ética" (Pérez, 2012).

\section{Antecedentes y estado de la cuestión}

Las huellas primigenias del A-S se sustentan en el aprendizaje experiencial (Dewey, 1967) y el servicio a la comunidad (James, 1910). En su configuración sociohistórica se diferencia de otras prácticas experienciales (voluntariado, trabajo de campo, actividades puntuales, etc.) por conectar los procesos "de aprendizaje y de servicio a la comunidad en un solo proyecto bien articulado en el que los participantes se forman al trabajar sobre necesidades reales del entorno con el objetivo de mejorarlo" (Puig, 2009, p. 29).

El estudio científico de la evolución de dicho fenómeno ha posibilitado "un consenso a nivel internacional que apunta a definir al aprendizaje-servicio a partir de tres rasgos fundamentales" (Montes, Tapia y Yaber, 2011, p. 10): el servicio a la comunidad con la intención de mejorarla, la participación activa de las personas implicadas y la planificación intencional de los objetivos curriculares y las acciones que conforman el servicio.

El A-S, como práctica pedagógica, se viene implementando en el ámbito internacional desde los años 70. En el territorio español su difusión se inicia a en los primeros años del siglo XXI a través de la Fundación Zerbikas en el País Vasco y el Centro Promotor de Aprendizaje-Servicio en Cataluña, realizando diversas actividades formativas dirigidas al profesorado de los diferentes niveles educativos y otros agentes socioeducativos, así como la sistematización y difusión de buenas prácticas, la presencia en la universidad, congresos, medios de comunicación, etc., y la creación de la Red Española de aprendizaje Servicio que integra a la Red Universitaria de Aprendizaje-Servicio (Mayor 
y Rodríguez, 2015). En la actualidad se ha implantado en los centros educativos de Cataluña como un servicio a la comunidad obligatorio dentro de los planes de estudio de la Educación Secundaria Obligatoria (Vallespín, 2014).

En relación a las prácticas de A-S implementadas, los hallazgos encontrados (Batlle, 2013; Furco, 2007; Puig, 2009) indican que constituyen un mosaico donde confluyen diversos propósitos, servicios, edades, ámbitos, instituciones, etc., encuadrados en un modelo estructural flexible (Véase Figura 1) que señala sus señas de identidad.

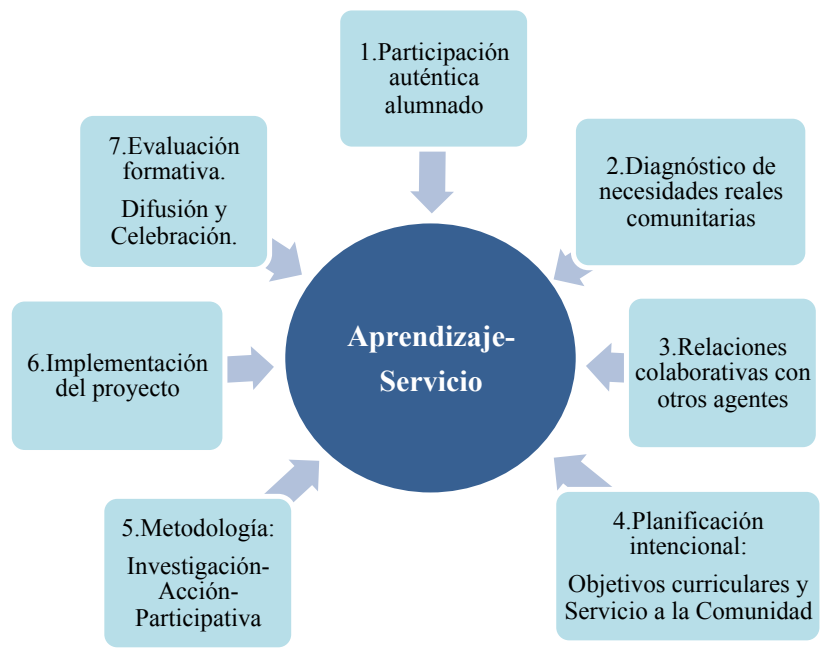

Figura 1. Elementos constitutivos de las prácticas de Aprendizaje-Servicio. Elaboración propia.

La gran heterogeneidad y profusión de acciones de A-S realizadas en la actualidad, ha provocado el interés y la necesidad de promover el estudio científico de las mismas. En esta dirección se han desarrollado distintas indagaciones en el contexto internacional (Furco, 2007; Conrad y Hedin, citados en Tapia, 2001; Herrero y Tapia, 2012) dirigidas a obtener datos rigurosos sobre el impacto producido en cuatro elementos significativos de los proyectos de A-S: estudiantes, docentes, escuelas universitarias y comunidad. Los resultados obtenidos evidencian los impactos positivos del A-S en los estudiantes en seis campos: "desarrollo académico y cognitivo; desarrollo cívico; desarrollo vocacional y profesional; desarrollo ético y moral; desarrollo personal y desarrollo social" (Furco, 2007, p. 177). También resaltan que los estudios sobre los otros tres elementos, anteriormente señalados, son todavía insuficientes para dar cuenta de esta práctica pedagógica de naturaleza multidimensional y multidisciplinar.

En este artículo indagamos en los cambios producidos en distintas dimensiones de la práctica docente como consecuencia de la puesta en marcha de un proyecto de A-S. La investigación que aquí se expone constituye una aportación novedosa en el ámbito español, pues no se conocen en nuestro país estudios sobre A-S focalizados en la temática que se aborda. 


\section{Metodología}

Para entender la singularidad del contexto donde se ha desarrollado la investigación, se describen dimensiones del mismo, para posteriormente abordar el enfoque metodológico.

\section{Contexto de la investigación}

El estudio se llevó a cabo en un centro educativo público, ubicado en un barrio socialmente desfavorecido, catalogado por las administraciones públicas como Zona con Necesidades de Transformación Social, debido a las situaciones de exclusión social que vive una parte significativa de la población (Junta de Andalucía, 2005). En este contexto se vienen implementado múltiples acciones socioeducativas de carácter comunitario desde el año 2006, donde participaban, de forma colaborativa, profesionales de diversos recursos públicos de la zona, entidades sociales y profesorado y alumnado de la universidad de Almería.

Durante el periodo 2012-2013 y 2013-2014, se constituyó, además, un grupo de investigación, aprobado por la Dirección General de Innovación Educativa y Formación del Profesorado, en el marco del Programa socioeducativo para la recuperación de El Ingenio-El Puche, integrado por docentes de distintos niveles educativos y profesionales de otros recursos públicos y entidades sociales, con el objetivo de desarrollar proyectos de A-S que promoviesen la adquisición aprendizajes útiles y significativos vinculados a la mejora de la realidad escolar y social (Mayor et al., 2012).

El proyecto elegido como objeto de indagación se realizó en un aula de $3^{\circ}$ primaria y tenía como propósito el diseño e implementación de una campaña de sensibilización para mejorar la limpieza de algunas zonas del barrio. La conformación y desarrollo del mismo, como proceso emergente, se describe en el apartado de resultados de la investigación.

\section{Enfoque metodológico}

De acuerdo con el objetivo de estudio, se optó por un enfoque metodológico de naturaleza cualitativa, ya que favorece la comprensión de la complejidad de los fenómenos sociales desde la perspectiva de sus actores (Pérez Juste, Galán y Quintanal, 2012), tratando de identificar la naturaleza profunda de las realidades, su estructura dinámica, aquella que da razón plena de su comportamiento y manifestaciones en contextos socio-históricos concretos (Guba, 1989).

Para aprehender el fenómeno educativo objeto de investigación, se utilizó el estudio de caso, con la intención de comprender y explicar la idiosincrasia y complejidad de un fenómeno singular (Simons, 2011; Stake, 2010): la implementación de una práctica pedagógica-didáctica nueva, en la cual la docente asumió el reto de comprometerse en una acción educativa que implicaba cambios en su forma habitual de pensar y desarrollar su quehacer profesional.

La investigación mediante estudio de caso sigue unas fases generales ampliamente aceptadas (Álvarez y San Fabián, 2012): a) Fase preactiva donde se explicitan los fundamentos epistemológicos, el objetivo de la investigación, selección de participantes, ins- 
trumentos de recogida de información, plan de trabajo, etc.; b) Fase interactiva o entrada en el campo para rastrear en los asuntos relacionados con el objetivo de la investigación a través de los distintos instrumentos de recogida de información previstos, etc.; c) Fase postactiva o final donde se analizan los datos y se prepara el informe del estudio.

\section{Participantes}

Los informantes, previo consentimiento negociado, han sido: 1 maestra y 17 alumnos de primaria, 5 alumnos y 1 profesora de la universidad y 1 representante de una entidad social. Para su selección se siguieron los siguientes criterios: obtener la máxima rentabilidad de aquello que aprendemos, la disponibilidad e interés de las personas implicadas para colaborar positivamente (Stake, 2010), así como el mayor tiempo de recorrido de la experiencia.

\section{Instrumentos y procedimiento}

El proceso de indagación se realizó a lo largo de un curso escolar 2012-13, para ello se utilizaron diversas técnicas cualitativas (Ruíz, 2003; Taylor y Bogdan, 1992) con diferentes propósitos, en distintos momentos y agentes implicados:

- Observación participante: en el aula y en las distintas actividades que, derivadas del proyecto, se realizaban en algunos lugares del barrio. Recogidas en notas de campo, han constituido el Diario de Observación del investigador, cuya permanencia en el campo era un día a la semana durante dos horas diarias.

- Entrevista semiestructurada grupal: se efectuaron dos, dirigidas al alumnado de primaria y de la universidad, con la intención de contrastar algunas informaciones obtenidas durante el proceso de observación, así como ampliar algunos asuntos que emergían durante la indagación.

- Entrevista semiestructurada individual: se realizaron dos a la presidenta de la Asociación de mayores y tres a la profesora de la universidad.

- Entrevista informal y entrevista semiestructurada individual dirigidas a la maestra: la primera se realizó en diez ocasiones con el propósito de profundizar en algunos aspectos concretos de interés para la investigación que iban emergiendo durante el proceso de observación. Se recogieron en el Diario de Observación. Para la segunda se configuró un guion abierto, validado según el procedimiento de juicio de expertos, con el propósito de enfocar las cuestiones relevantes para el objetivo de la investigación.

- Análisis de documentos: se centró en la Programación Curricular de Aula, Proyecto Educativo de Centro, Programa de la asignatura de Innovación Educativa, Diario de prácticas del alumnado de la Universidad, materiales didácticos elaborados por el estudiantado de primaria, etc.

Para el registro de las observaciones y de las entrevistas en profundidad se utilizó el vídeo, y se realizó la transcripción literal de todo lo expresado y acontecido con el propósito de eliminar el sesgo del investigador e intentar asegurar la credibilidad y dependencia o replicabilidad de los datos (Guba, 1989; Álvarez y San Fabián, 2012). 


\section{Análisis de los datos}

Los datos obtenidos fueron analizados siguiendo la técnica de análisis de contenido, con el interés de configurar las categorías más relevantes. Para ello se llevó a cabo un conjunto de tareas de comparación, contrastación, agregación y ordenación (Simons, 2011) sobre los datos, que permitieron identificar las unidades de significado, codificar las mismas y posteriormente conformar las siguientes categorías:

Tabla 1

Categorías, subcategorías y códigos fijados en el análisis

\begin{tabular}{|c|c|c|c|c|c|}
\hline Categorías & Códigos & Subcategorías & Códigos & Subcategorías & Códigos \\
\hline \multirow{3}{*}{$\begin{array}{l}\text { Concepciones } \\
\text { pedagógicas }\end{array}$} & \multirow{3}{*}{$\mathrm{CP}$} & $\begin{array}{l}\text { Sentido de la } \\
\text { educación. }\end{array}$ & CPSE & Rol profesional. & CPRP \\
\hline & & Programación flexible. & $\mathrm{CPPF}$ & $\begin{array}{l}\text { Relaciones } \\
\text { colaborativas. }\end{array}$ & CPRC \\
\hline & & Ideas A-S. & CPAS & $\begin{array}{l}\text { Intereses } \\
\text { pedagógicos. }\end{array}$ & CPIP \\
\hline \multirow{5}{*}{$\begin{array}{l}\text { Programación } \\
\text { de aula }\end{array}$} & \multirow{5}{*}{ PA } & $\begin{array}{l}\text { Adaptación del } \\
\text { currículum al servicio } \\
\text { a la comunidad. }\end{array}$ & PAAC & $\begin{array}{l}\text { Colaboración con } \\
\text { otros agentes. }\end{array}$ & PACA \\
\hline & & & & Reflexión-acción. & PARA \\
\hline & & $\begin{array}{l}\text { Contextualización de } \\
\text { contenidos. }\end{array}$ & PACC & Rol docente. & PARD \\
\hline & & $\begin{array}{l}\text { Participación } \\
\text { alumnado. }\end{array}$ & PAPA & $\begin{array}{l}\text { Creación materiales } \\
\text { didácticos. }\end{array}$ & PAMD \\
\hline & & $\begin{array}{l}\text { Actividades dentro y } \\
\text { fuera del aula. }\end{array}$ & PAAD & $\begin{array}{l}\text { Reorganización } \\
\text { de tiempos y } \\
\text { actividades. }\end{array}$ & PART \\
\hline \multirow{3}{*}{$\begin{array}{l}\text { Desarrollo } \\
\text { profesional }\end{array}$} & \multirow{3}{*}{ DP } & $\begin{array}{l}\text { Nuevos retos } \\
\text { profesionales. }\end{array}$ & DPRP & $\begin{array}{l}\text { Relaciones con } \\
\text { otros agentes. }\end{array}$ & DPRA \\
\hline & & $\begin{array}{l}\text { Aprendizaje contenido } \\
\text { nuevo (A-S). }\end{array}$ & DPAC & $\begin{array}{l}\text { Mayor } \\
\text { conocimiento del } \\
\text { barrio. }\end{array}$ & DPCB \\
\hline & & $\begin{array}{l}\text { Iniciar proyectos de } \\
\text { trabajo. }\end{array}$ & DPTP & $\begin{array}{l}\text { Satisfacción } \\
\text { profesional y } \\
\text { personal. }\end{array}$ & DPSP \\
\hline
\end{tabular}

Fuente: elaboración propia.

Con la intención de obtener la credibilidad o validez interna, así como la confirmabilidad y la transferibilidad de los datos alcanzados (Guba, 1989; Taylor y Bogdan, 
1992), se llevaron a cabo distintas estrategias: a) contextualización de la unidad de análisis; b) registro audiovisual de la información obtenida; c) negociación de las transcripciones con las personas implicadas, y d) la triangulación entre los diversos instrumentos de recogida de información y entre las disímiles fuentes de información utilizadas:

Tabla 2

Relación de informantes y técnicas de recogida de información

\begin{tabular}{lcccc}
\hline & $\begin{array}{c}\text { Observación } \\
\text { participante }\end{array}$ & $\begin{array}{c}\text { Entrevista } \\
\text { individual }\end{array}$ & $\begin{array}{c}\text { Entrevista } \\
\text { grupal }\end{array}$ & $\begin{array}{c}\text { Análisis } \\
\text { documentos }\end{array}$ \\
\hline Maestra primaria (1) & $X$ & $X$ & & \\
\hline Alumnado primaria (17) & $X$ & & & \\
\hline Profesora universidad (1) & $X$ & $X$ & $X$ \\
\hline Alumnado universidad (5) & $X$ & & \\
\hline Representante entidad social (1) & $X$ & $X$ & \\
\hline
\end{tabular}

Fuente: elaboración propia.

Por tanto, la información expuesta en el siguiente epígrafe es el resultado de contrastar permanentemente los asuntos de interés que se iban obteniendo a través de los diferentes instrumentos de recogida de datos.

\section{Resultados}

Los resultados de tipo cualitativo se expresan de manera narrativa, sistematizados en tres categorías interrelacionadas, con descripciones de los temas más significativos que sirvieron su conformación.

\section{Concepciones pedagógicas}

La práctica educativa es una realidad dinámica constituida por diversas dimensiones: valores, modos de hacer y formas de pensar, conocimientos implícitos y explícitos, componentes racionales y emocionales, contexto sociocultural donde se implementan, etc., donde las ideas pedagógicas juegan un papel sustantivo en la intencionalidad y orientación de la misma (Escudero, 2009). Desde esta perspectiva, la implicación de la maestra en el grupo de investigación, descrito anteriormente, la situó ante "un nuevo reto profesional" (Ent. $\mathrm{M}^{1}$.) que conllevaba cambios significativos relacionados con las distintas dimensiones de su práctica habitual anterior, orien-

1 Abreviaturas utilizadas para referirnos a los y las diferentes informantes y técnicas de recogida de datos: Ent. M. (Entrevista Maestra); Ent. A. U. (Entrevista Alumnado Universidad); Ent. P. U. (Entrevista Profesora Universidad); Ent. A. P. (Entrevista Alumnado Primaria); Ent. P. A. M. (Entrevista Presidenta Asociación Mayores); D. O. (Diario Observación). 
tada, fundamentalmente, hacia la adquisición de conocimientos descontextualizados y con escaso valor de uso que proponen los libros de texto. Quebrar esta dinámica, no estaba exenta de dificultades: "Al principio, trabajar sin libros de texto, era como conducir sin manos" (Ent. M.).

Las nuevas propuestas de trabajo que emergían en el espacio del grupo de investigación tenían como eje articulador de reflexión-acción los proyectos de A-S. En este escenario complejo, las ideas pedagógicas previas de la docente y de otros profesionales se fueron confrontando y debatiendo, produciéndose distorsiones, situaciones problemáticas y posibilidades de acción conjunta, que requerían de la deliberación de la docente para posicionarse ante las mismas (D.O.).

En este caso, la maestra concebía el A-S como "una práctica educativa para que los menores aprendan prestando un servicio a su barrio y tomen conciencia de que ellos pueden hacer algo por el mismo" (Ent. M.). Esta dimensión social de la educación es una de las cuestiones que suscita su interés inicial, junto con la posibilidad de "trabajar colaborativamente con el alumnado y profesorado de la universidad, otros agentes socioeducativos del barrio, y el aprendizaje de nuevos conocimientos" (Ent. M.).

Se abría así a un nuevo espacio de posibilidad que requería repensar las acciones alejada de las prescripciones de los libros de texto, para explorar, junto a otros agentes educativos, nuevas formas de pensar y situarse ante el hecho educativo, más próximas a lo que Schön (1992) denomina como profesional reflexivo. Su implicación en el diseño del proyecto de A-S la condujo a sustentar la programación didáctica en función del "servicio a la comunidad decidido por los menores" (D.O.), a reconfigurar su práctica profesional y asumir su responsabilidad y protagonismo, junto a otros actores, en el diseño de un proyecto educativo "participativo, flexible y contextualizado" (Ent. M.) donde el conocimiento era valorado por su utilidad personal y social, ya que "tiene que servir para alcanzar los propósitos planteados" (Ent. M.).

En esta articulación emergente, donde los objetivos curriculares y los derivados del servicio a la comunidad se retroalimentan mutuamente, "la reflexión en y sobre la práctica se convierte en el eje transversal que orienta las distintas actividades, ya que el conocimiento no se presenta como acabado" (Ent. M.), sino como una construcción social situada y dinámica.

Esta nueva forma de afrontar el hecho educativo, era resaltada por el alumnado de la universidad:

Esta experiencia me ha marcado, ya que he visto otras formas de hacer las cosas. Por ejemplo: la visita que hicimos al río para ver cómo se encontraba y realizar juegos de sensibilización medioambiental, la aprovechamos para trabajar la historia del barrio, ver lo que los menores conocían del mismo y después trabajar esa información en distintas áreas curriculares (Ent. A. U.5). 
Por su parte, la profesora de la universidad, responsable del seguimiento de las prácticas del estudiantado de su asignatura que participaba en el proyecto, comentaba:

En la exposición que ha realizado la maestra en la sesión del grupo de investigación y lo que he observado las tres veces que he visitado el aula, me ha parecido muy potente su capacidad y osadía para pensar, actuar y atreverse con un proyecto nuevo. Pensar en la experiencia de la maestra, me ha hecho pensar en mi relación con el alumnado de mi asignatura (Ent. P.U.).

\section{Programación aula}

El proyecto de A-S se ha ido articulando en los espacios de intersección donde confluían, por un lado, la planificación intencional de los aprendizajes curriculares y las actividades asociadas al servicio a la comunidad y, por otro, la emergencia de nuevas propuestas realizadas por distintos participantes y las singularidades del contexto donde se implementaban (D.O.).

El proceso anterior de análisis de la propia práctica sirvió a la docente para cambiar concepciones pedagógicas asentadas y entender el curriculum como un campo de experimentación e indagación (Santos Guerra, 2010) a partir de problemas reales que surgen en las aulas y otros espacios por donde circula la educación. El curriculum como praxis (Stenhouse, 2007) supone interpretarlo como proyecto en ejecución que se verifica en la acción del aula, posibilitando que la programación de aula y sus elementos constitutivos vayan reformulándose sobre la propia acción didáctica.

En cuanto a la incidencia del proyecto de A-S en la programación de aula se ha podido constatar una influencia significativa en diferentes elementos:

Tabla 3

Cambios significativos producidos en la Programación Curricular Aula

\begin{tabular}{lll}
\hline $\begin{array}{c}\text { Dimensiones } \\
\text { curriculares }\end{array}$ & $\begin{array}{c}\text { Antes de la implementación } \\
\text { del proyecto de A-S }\end{array}$ & $\begin{array}{c}\text { Durante la implementación del } \\
\text { proyecto de A-S }\end{array}$ \\
\hline Contenidos & Libro de texto. & $\begin{array}{l}\text { Distintas fuentes relacionadas con } \\
\text { el contexto y el servicio a la comu- } \\
\text { nidad. }\end{array}$ \\
$\begin{array}{ll}\text { Materiales } \\
\text { didácticos }\end{array}$ & $\begin{array}{l}\text { Libro de texto, canciones, cuen- } \\
\text { tos, etc. }\end{array}$ & $\begin{array}{l}\text { Labreta de mi vida, maquetas, } \\
\text { tipi, carteles, entrevistas, cancio- } \\
\text { nes, libro de texto, etc. }\end{array}$ \\
Actividades & Propuestas libro de texto. & $\begin{array}{l}\text { Relacionadas con el servicio: iti- } \\
\text { nerarios de investigación por el } \\
\text { barrio, entrevistas a comerciantes } \\
\text { y otros profesionales de recursos } \\
\text { públicos, creación de maquetas, } \\
\text { carteles, etc. }\end{array}$ \\
& &
\end{tabular}




\begin{tabular}{|c|c|c|}
\hline Metodología & $\begin{array}{l}\text { Transmisiva, trabajo individual } \\
\text { y gran grupo. }\end{array}$ & $\begin{array}{l}\text { Colaborativa, trabajo en pequeños } \\
\text { grupos, asamblea e individual. }\end{array}$ \\
\hline $\begin{array}{l}\text { Relación maestra- } \\
\text { alumnado }\end{array}$ & Cercana, respetuosa, afectiva. & $\begin{array}{l}\text { Participación de otros agentes } \\
\text { educativos, cercana, respetuosa, } \\
\text { afectiva. }\end{array}$ \\
\hline Espacios & Aula-centro educativo. & Aula-centro educativo-barrio. \\
\hline $\begin{array}{l}\text { Temporalización } \\
\text { actividades }\end{array}$ & 45 minutos. & 45 y/o 90 minutos. \\
\hline Evaluación & Resultados. & Proceso y resultados. \\
\hline
\end{tabular}

Fuente: elaboración propia.

La interacción permanente entre los objetivos inicialmente previstos y las nuevas dificultades y posibilidades que iban emergiendo como "consecuencia de la reconstrucción de aprendizajes de los menores" (Ent. M.), así como de "las propuestas que iban realizando los menores, el alumnado de la universidad y otros agentes socioeducativos" (Ent. M.), condujeron a la docente a trabajar con una "programación curricular flexible que se concretó en un plan de acción que aprobamos en una asamblea" (Ent. M.).

La selección de contenidos vinculados con el servicio "que había elegido el alumnado, me llevó a contemplar los libros de texto como un recurso didáctico más, ya que no contenían información valiosa para el objetivo planteado" (Ent. M.). Esto constituyó un desarrollo de las capacidades reflexivas y creativas de la docente y el alumnado de primaria y de la universidad:

Para el diseño e implementación del servicio: “Campaña de sensibilización para mejorar la limpieza del barrio" tuvimos que utilizar y crear distintas fuentes provisoras de información relacionadas con la vida en el barrio: historia, calles, instituciones públicas, entidades sociales, características de la población, etc. (Ent. M.).

Para adquirir conocimientos sobre dichos asuntos se utilizaron, entre otros, los siguientes materiales didácticos:

Noticias de televisión, internet, canciones, elaboración y realización de entrevistas a personas significativas del barrio, fichas de investigación y plan de acción, libros de texto, maquetas, historias del barrio contadas por las vecinas, etc. (Ent. M.).

Muchas de las actividades contempladas en el servicio requerían "traspasar los límites del centro y pensar en el barrio como escenario educativo" (Ent. M.). En el desarrollo de las mismas se podía observar la "conexión entre las realizadas en el contexto de aula y las implementadas en el barrio" (D.O.):

Realizamos una salida al río, acompañados por el alumnado de la universidad. Aprovechamos el trayecto para investigar distintos elementos del barrio 
y ver la zona donde se realizaría la campaña de sensibilización. Los datos recogidos y la experiencia vivida la evaluamos posteriormente en la asamblea de clase. Durante el debate surgieron dudas relacionadas con la acumulación de basura, los posibles problemas de salud y los horarios y lugares de la recogida de basura y otros enseres [...]. Decidí contactar con profesionales del centro de salud, de la empresa municipal de limpieza y con la presidenta de la asociación de mayores, para que el alumnado resolviera sus dudas. Parte de la información recogida en estas actividades, la utilizamos para diseñar el cartel de la campaña de sensibilización (Ent. M.).

Para realizar las actividades en el barrio, la maestra modificó los tiempos asignados a algunas materias curriculares, "ya que 45 minutos no son suficientes para llevar a cabo las mismas" (Ent. M.).

El servicio a la comunidad requería del establecimiento de relaciones colaborativas entre distintos agentes para su diseño e implementación:

Para que los menores obtengan informaciones que le ayuden a sostener con más razón sus propuestas, he decidido solicitar la colaboración de los profesionales del centro de salud, para que nos informen de las posibles enfermedades que puede acarrear la acumulación de basura en algunos espacios públicos, y qué medidas de prevención podemos adoptar (Ent. M.).

Y por otro lado, reconocer y aprovechar el potencial educativo que pueden aportar al proceso formativo del estudiantado:

Durante la visita que realizaron a la empresa municipal de limpieza, los menores recogieron información sobre el reciclaje de distintos elementos: papel, plástico, pilas, aceite, etc., y sobre los horarios de recogida de basura y otros enseres (Ent. M.).

En esta línea, el alumnado de primaria exponía:

Hemos aprendido que la basura hay que echarla dentro del contendor porque si no acuden las ratas y pueden provocar enfermedades (Ent. A.P.5).

Que los electrodomésticos los tenemos que colocar al lado del contenedor de basura y no echarlos al río (Ent. A.P.3).

También "le pedimos a la presidenta de la asociación de mayores del barrio que viniera a clase para contarnos el origen del barrio y cómo ha ido evolucionando. Aprovechamos su visita para que los menores expusieran la campaña de sensibilización que estaban llevando a cabo" (Ent. M.).

En línea con lo anterior, la presidenta de la asociación de mayores planteaba: “Desde que los niños fueron a hablar con los comerciantes y pegaron los carteles, esa zona del barrio está más limpia" (Ent. P.A.M.). 
La evaluación del proceso, considerada como eje transversal del conjunto de actividades, "me dio pistas para ver en cada momento cómo estaban viviendo cada uno de los menores el proyecto y para incorporar nuevos elementos que iban apareciendo en el transcurrir del mismo" (Ent. M.).

Como síntesis de su tránsito por esta experiencia exponía: "ha sido muy positiva, ya que los menores vienen contentos a clase y, para mí, como profesional y como persona, no hay nada mejor que los niños y las niñas vengan con ganas de aprender" (Ent. M.).

\section{Desarrollo profesional}

El A-S como práctica educativa implica un ejercicio de reconstrucción de los saberes, ya que enfrenta a los y las participantes a problemas sociales y educativos que están conectados a diversas perspectivas y posiciones en el abordaje de los mismos. En este sentido, la implicación inicial de la docente en el proyecto de A-S fue vivida "como un reto profesional y personal que me ha permitido trabajar de forma diferente e integrar las distintas áreas curriculares en la programación de aula" (Ent. M.).

Pensar su quehacer profesional desde una propuesta educativa que suponía cambios significativos en los marcos de referencia que sustentaban su práctica anterior, provocó la reflexión de la docente y un cambio sustancial en su forma de entender la programación de aula, ya que ésta pasó de estar orientada, fundamentalmente, por los libros de texto, a enfocarse en los aprendizajes necesarios para realizar una actividad educativa de utilidad social en un contexto real donde participaban diferentes agentes socioeducativos.

Un salto cualitativo importante se ha venido produciendo en una dimensión del desarrollo profesional: la formación permanente del profesorado implicado en este proyecto, efectuada de manera contextualizada en el propio centro donde el proyecto se ponía en acción. Ha supuesto, además, una reflexión que va más allá de los aspectos puramente técnicos, abordando cuestiones éticas, relacionales, colaborativas, etc.

Su interés por la dimensión social del proyecto: "estoy intentando que los menores entiendan que pueden cambiar algo del barrio" (Ent. M.), y su compromiso profesional, la llevó a investigar en distintas publicaciones sobre "un tema (A-S) del que no conocía nada" (Ent. M.). Los conocimientos adquiridos en el proceso, junto a "las respuestas positivas del alumnado de primaria y el sentirme acompañada por el alumnado y profesorado de la universidad, me ayudó a diseñar una programación de aula pensando en el aprendizaje que los menores podían obtener a través de la realización de acciones dentro y fuera del aula" (Ent. M.). En esta línea de pensamiento la profesora de la universidad relataba: "En la medida que la maestra vaya tratando de concienciar a los menores de que pueden cambiar su realidad, ella también se irá concienciando de su realidad, de sus carencias y las tendrá que ir afrontando" (Ent. P.U.).

Concebir su práctica profesional desde otros parámetros de la educación ("Los menores aprenden prestando un servicio a la comunidad". Ent. M.) la condujo a indagar en el territorio, para obtener información de los recursos públicos y entidades sociales que podían participar en el proyecto. Dicha actividad la llevó a conectar y concretar la realización de actividades conjuntas y, por otro lado, le aportó "un mayor conocimiento del barrio" (Ent. M.). 
Como síntesis de sus aprendizajes en esta práctica pedagógica, la docente expresaba su satisfacción con el proceso vivido:

Esta experiencia ha sido muy gratificante y enriquecedora, ya que me ha posibilitado conocer más la realidad de los menores, adaptar la programación de aula a sus intereses, aprender a trabajar con proyectos colaborativos y el contenido de un tema nuevo (A-S) del que no tenía ni idea (Ent. M.).

\section{Conclusiones y discusión}

Atendiendo a los resultados alcanzados, se puede concluir que, en términos generales, los proyectos de A-S, en su versión fuerte (Díaz, 2006), tienen un impacto positivo en diversos elementos de la práctica docente: concepciones pedagógicas, diseño y puesta en acción de la programación didáctica y desarrollo profesional del profesorado.

En primer lugar, los hallazgos encontrados ponen de manifiesto que la implicación de la docente en las práctica de A-S ha supuesto una reconfiguración progresiva de sus concepciones pedagógicas, situadas inicialmente, en lo que Carr (1996), denomina racionalidad técnica o instrumental, donde el profesorado se piensa como un técnico que suministra al alumnado conocimientos elaborados por expertos, sin tener en cuenta el contexto social y la significatividad y utilidad para la vida del alumnado; se han ido reconstruyendo al comprometerse con una acción pedagógica situada de carácter reflexivo que conecta con sus intereses pedagógicos y supone otra forma de entender el quehacer docente, siendo la reflexión-acción el eje promotor del proceso de construcción de aprendizajes significativos, útiles y relevantes orientados a mejorar aspectos de la realidad escolar y social.

Esta nueva forma de pensar, hacer y relacionarse está motivada, entre otras cuestiones, por la apertura de un escenario de posibilidad donde confluye el interés y el compromiso de la docente, así como las ideas nucleares y elementos que conforman los proyectos de A-S, ya que ofrecen un contexto real de investigación-acción en el cual el profesorado, y otros agentes, tienen un rol protagónico en su diseño, implementación y evaluación. Esta apreciación coincide con algunas investigaciones realizadas (Díaz, 2006; Furco, 2007; Jouannet, Ponce y Contreras, 2012) donde se confirma que los docentes que participan en la práctica de A-S cambian su concepción de la enseñanza, así como la percepción de los mismos en distintas dimensiones de su quehacer docente: incremento de la motivación, mejora significativa de la relación profesor-alumno, disposición para incorporar nuevas innovaciones, etc.

En segundo lugar, los resultados obtenidos también evidencian que las prácticas de A-S se estructuran y funcionan como dispositivos complejos que conllevan cambios sustantivos en los diferentes elementos que integran la programación curricular de aula diseñada, en sus orígenes, desde una racionalidad instrumental o técnica. La interacción permanente entre los objetivos curriculares y los previstos en el servicio a la comunidad van conformando un circulo virtuoso (Montes et al., 2011), ya que los aprendizajes académicos mejoran el servicio ofrecido, y el servicio estimula la adquisición o producción de nuevos conocimientos para dar respuestas adecuadas a 
la necesidad detectada. En esta línea, y en consonancia con las aportaciones teóricas de diferentes autores (Pérez, 2012; Puig, Gijón, Martín y Rubio, 2011), este estudio señala la bondad pedagógica de los proyectos de A-S para configurar prácticas reflexivas y críticas, estructuradas por guiones curriculares flexibles donde los objetivos curriculares y los derivados del servicio a la comunidad van conformando una red de vasos comunicantes que se retroalimentan mutuamente.

En tercer lugar, los logros alcanzados sirven para constatar que las acciones pedagógicas de A-S requieren de una implicación fuerte del profesorado en las distintas fases de su puesta en acción, lo que favorece el desarrollo personal y profesional de los docentes, ya que esta acción deliberada y situada (Schön, 1992) resquebraja las formas tradicionales de entender la práctica docente configurada, entre otros elementos, por la transmisión de conocimientos descontextualizados y con escaso valor de uso, para ir articulándose en un guion flexible, donde las distintas dimensiones que lo configuran no están dadas de antemano, sino que son construidas a partir de situaciones concretas que requieren una actitud investigativa para intentar dar respuestas creativas a las inquietudes y problemáticas que van emergiendo en un contexto real.

Formularse replanteamientos desde otras perspectivas que requieren posiciones alternativas para afrontar una nueva práctica pedagógica-didáctica, le ha posibilitado a la docente la adquisición de aprendizajes múltiples: “Esta experiencia me ha servido para aprender con otras personas, descubrir un contenido nuevo, trabajar por proyectos..." (Ent. M). En esta indagación se ha comprobado el protagonismo reflexivo y crítico de la docente, así como la influencia de las prácticas de A-S en la reconstrucción de sus saberes.

De acuerdo con lo anteriormente expuesto, así como con algunas investigaciones realizadas (Díaz, 2006; Furco, 2007; Root, Callagan y Sepansky, 2012, citados en Aramburuzabala y García, 2013) y con las propuestas de mejora para transformar la educación que plantean distintos teóricos (Hargreaves y Fullan, 2014; Pérez, 2012; Schön, 1992) de las ciencias sociales, consideramos que las prácticas pedagógicas de A-S pueden ser concebidas como escenarios formativos que favorecen el desarrollo profesional.

En relación a los límites de este estudio y su posible aportación a la comunidad científica, cabe señalar que una limitación de esta indagación es su carácter de estudio de caso a partir de un fenómeno singular, por lo que no se pretende la generalización de los resultados a otros casos, aunque los resultados se pueden transferir de un contexto a otro, dependiendo del grado de similitud entre los dos contextos (Guba, 1989; Pérez Juste et al., 2012) y, por otro lado, pueden significar "una oportunidad nueva de modificar las antiguas generalizaciones "(Stake, 2010, p. 78).

En lo que concierne a la prospectiva, se sugiere avanzar en distintas líneas:

- Explorar la influencia de esta experiencia en la formación del alumnado de primaria y de la universidad a medio-largo plazo.

- Indagar las razones que sustentan la participación de los distintos agentes implicados.

- Estudiar el tipo y niveles de participación que promueven los proyectos en menores y jóvenes. 
- Analizar la influencia de los proyectos de A-S en los cambios y mejoras producidas en el centro educativo y en la comunidad.

\section{Referencias bibliográficas}

Álvarez, C. y San Fabián, J. L. (2012). La elección del estudio de caso en investigación educativa. Gazeta de Antropología, 28(1), 1-12. Recuperado de http://www.ugr. es/ pwlac/G28_14Carmen_Alvarez-JoseLuis_SanFabian.html

Aramburuzabala, P. y García, R. (2013). Aprendizaje-Servicio en la formación de maestros comprometidos con la justicia social. En L. Rubio, E. Prats y L. Gómez (Coord.). Universidad y sociedad. Experiencias de aprendizaje servicio en la universidad (pp. 14-21). Barcelona: Universitat de Barcelona. Recuperado de http://hdl.handle. net/2445/46344

Batlle, R. (2013). Aprendizaje-Servicio en España: el contagio de una revolución pedagógica necesaria. Madrid: PPC.

Carbonell, J. (2001). La aventura de innovar. El cambio en la escuela. Madrid: Morata.

Carr, W. (1996). Una teoría para la educación. Hacia una investigación educativa crítica. Madrid: Morata.

Delors, J. (coord.). (1996). La educación encierra un tesoro. Madrid: Santillana.

Dewey, J. (1967). El niño y el programa escolar: mi credo pedagógico. Buenos Aires: Losada.

Díaz, F. (2006). Enseñanza situada: vínculo entre la escuela y la vida. México: McGranHill Interamericana.

Escudero, J. M. (2009). Buenas prácticas y programas extraordinarios de atención al alumnado en riesgo de exclusión educativa. Profesorado. Revista de currículum y formación del profesorado, 13(3), 107-141. Recuperado de http://www.ugr. es/ recfpro/rev133ART4.pdf

Faure, E. (1972). Aprender a ser. Madrid: Alianza.

Furco, A. (2007). Impacto de los proyectos de aprendizaje-servicio. En J.C. Tedesco, I. Hernaiz,

M. Nieves, S. Rial. (Eds.), Antología 1997-2007. Seminarios Internacionales “Aprendizaje y Servicio Solidario" (pp. 175-183). Buenos aires: EUDEBA Recuperado de http:// repositorio.educacion.gov.ar/dspace/bitstream/handle/123456789/94942/2007_antologia_10.pdf?sequence=1.

García, T. y Carmona, J. J. (2014). Profesor y alumnado conectados a una red pública compartida. Revista de Investigación Educativa, 32(2), 463-478. http://dx.doi. org/10.6018/rie.32.2.171801

Giordan, A. y De Vecchi, G. (1995). Los orígenes del saber. Sevilla: Díada.

Guba, E. (1989). Criterios de credibilidad en la investigación naturalista. En J. Gimeno y A. Pérez. (Coords.). La enseñanza: su teoría y su práctica (pp. 148-165). Madrid: Akal. Hargreaves, A. y Fullan, M. (2014). Capital profesional. Madrid: Morata.

Herrero, M. A. y Tapia, M. N. (2012). Actas de la II Jornada de Investigadores sobre Aprendizaje-Servicio, 22 de agosto. Buenos Aires: CLAYSS. Recuperado de http:// www.clayss.org.ar/06_investigacion/jornadas/Libro_IIJIA-S_COMPLETO.pdf. 
Imbernón, F. (2011). Un nuevo desarrollo profesional del profesorado para una nueva educación. Revista de Ciencias Humanas, 12(19), 75-86.

James, W. (1910). El equivalente moral de la guerra. Traducción castellana de Mónica Aguerri. Recuperado de http://www.unav.es/gep/TheMoralEquivalentOfWar.html

Jouannet, C., Ponce, C. y Contreras, A. (2012). Impacto de la metodología de aprendizajeservicio $(A+S)$ según la percepción de los docentes. En M.A. Herrero y M.N. Tapia (Eds.), Actas de la II Jornada de investigadores en aprendizaje-servicio. Buenos Aires: CLAYSS.

Junta de Andalucía (2005). Actuaciones en Barriadas y Zonas con Necesidades de Transformación Social. Consejería para la Igualdad y el Bienestar Social.

López-Vargas, B. y Basto-Torrado, S. (2010). Desde las teorías implícitas a la docencia como práctica reflexiva. Educación y Educadores, 13 (2), 275-291.

Luengo, F. y Moyá, J. (2008). Escuela, familia, comunidad: claves para la acción. España: Wolters Kluwer.

Mayor, D., García, T., Sánchez, M. S., Rodríguez, D., Zaballos, Ma․ M., Pérez, I. “y” Romera, A. (2012). Programa socioeducativo de carácter comunitario para la recuperación de “El Ingenio-El Puche". C. Beltrán (coord.), I Edición concurso de proyectos de Educación Social (pp. 13-59). Barcelona: CGCEES-Consejo General de Colegios de Educadoras y Educadores Sociales.

Mayor, D. y Rodríguez, D. (2015). Aprendizaje-Servicio: construyendo espacios de intersección entre la escuela-comunidad-universidad. Profesorado. Revista de currículum y formación del profesorado, 19(1), 262-279.

Montes, R., Tapia, M. y Yaber, L. (2011). Manual para docentes y estudiantes solidarios. Buenos Aires: Natura-CLAYSS.

OCDE (2005). La definición y selección de competencias clave. Resumen ejecutivo. Recuperado de http://www.oecd.org/pisa/35070367.pdf

Pérez, A. I. (2012). Educarse en la era digital. Madrid: Morata.

Pérez Juste, R., Galán, R. y Quintanal, J. (2012). Métodos y diseños de investigación en educación. España: UNED- Universidad Nacional de Educación a Distancia.

Puig, J. M. (Coord.). (2009). Aprendizaje Servicio. Educación y compromiso cívico. Barcelona: Graó.

Puig, J. M., Gijón, M., Martín, X. y Rubio, L. (2011). Aprendizaje-servicio y Educación para la Ciudadanía. Revista de Educación, núm. ext., 45-67. Recuperado de http:// www.revistaeducacion.educacion.es/re2011/re2011_03.pdf

Ruíz, J. I. (2003). Metodología de la investigación cualitativa. Bilbao: Universidad de Deusto.

Santos, M.A. (2010). La formación del profesorado en las instituciones que aprenden. Revista Interuniversitaria de Formación del Profesorado, 68(24,2), 175-200.

Schön, D. (1992). La formación de profesionales reflexivos. Barcelona: Paidós.

Simons, H. (2011). El estudio de caso: Teoría y práctica. Madrid: Morata.

Stake, R.E. (2010). Investigación con estudio de casos (5 $5^{\mathrm{a}}$ ed.). Madrid: Morata.

Stenhouse, L. (2007). Investigación y desarrollo del curriculum (6 $6^{\underline{a}}$ edición). Madrid: Morata.

Tapia, M. N. (2001). La solidaridad como pedagogía. Buenos Aires: Ciudad Nueva. 
Taylor, S.J. y Bogdan, R. (1992). Introducción a los métodos cualitativos de investigación. Barcelona: Paidós.

Torres, J. (2011). La justicia curricular. El caballo de Troya de la cultura escolar. Madrid: Morata.

Vallespín, I. (2 de julio de 2014). Los alumnos de la ESO catalanes deberán realizar servicios a la comunidad. El País.

Fecha de recepción: 30 de junio de 2015.

Fecha de revisión: 30 de junio de 2015.

Fecha de aceptación: 29 de marzo de 2016. 